\title{
Dominant Coupling Mechanism for Integrated Circuit Immunity of SOIC Packages up to $10 \mathrm{GHz}$
}

\author{
Sjoerd Op 't Land ${ }^{* \dagger}$, Member, IEEE, Mohamed Ramdani*†, Senior Member, IEEE, Richard Perdriau*†, Senior \\ Member, IEEE \\ *RF-EMC Research Group, ESEO-TECH, \\ Angers, France \\ Email: s.t.optland@gmail.com, \{richard.perdriau, mohamed.ramdani\}@eseo.fr \\ ${ }^{\dagger} \mathrm{ADH}, \mathrm{IETR}$ \\ Rennes, France
}

\begin{abstract}
As the frequency of functional signals and interfering fields is rising beyond $1 \mathrm{GHz}$, the immunity of integrated circuits (ICs) against these higher frequencies is interesting. To design test setups that mimic the real-world interference an IC may receive, the dominant coupling mechanism (radiation or conduction) needs to be known. We hypothesize that the dominant coupling mechanism is conduction for SOIC packages up to about $10 \mathrm{GHz}$. To challenge this hypothesis, the radiated immunity of a Printed Circuit Board (PCB) trace connected to a voltage regulator $\mathrm{IC}$ is predicted and measured. The radiated immunity is predicted to be the product of the fieldto-trace attenuation and the conducted immunity of the IC, thus neglecting the radiated immunity of the IC. As far as could be measured, the prediction correlated well with measurement, so the dominant-conduction hypothesis was not falsified with this case study.
\end{abstract}

Index Terms-EMC, immunity, integrated circuit, modeling, GDPI, ICIM-CI, ICIM-RI, GTEM, ROME

\section{INTRODUCTION}

Electromagnetic Compatibility (EMC) problems may first appear in design, subsystem test, product test or after sales. The later problems surface, the costlier it is to solve them. Therefore, an early detection of immunity problems is interesting.

To that end, models are needed that are sufficiently precise to predict immunity problems. They should not be too simple, lest immunity problems be overlooked. They should not be too precise either, because that makes model extraction and usage more costly. To summarize, the models should have a positive Return On Modeling Effort (ROME).

One attempt at such a model is the Integrated Circuit Immunity Model for Conducted Immunity (ICIM-CI), which can be extracted from measurements on the IC to-be-modeled. It is standardized by the International Electrotechnical Commission (IEC) as standard 62433-4 [1].

It is interesting to note that these model and measurement techniques are (being) standardized by the IEC up to $1 \mathrm{GHz}$. This suggests that the model has a good ROME: real-life, industrial immunity problems can be predicted and explained sufficiently with a reasonable measurement and modeling effort, at least up to $1 \mathrm{GHz}$. Its radiated immunity counterpart (ICIM-RI), is not yet standardized, however. Of course, it might be a very hard problem, so there is no standard, despite a significant effort. However, the authors suspect there is no significant effort, because it is not yet necessary for industry to model the radiated immunity of the IC itself to obtain sufficient system immunity predictions.

The explanation seems obvious: as long as the IC package (hence each interconnect) is small with respect to the interference wavelength, the disturbance voltage gathered by the interconnects is small. Given that the size of ICs is in the order of centimeters, direct coupling of an interfering field to an IC can be neglected up to several $\mathrm{GHz}$ [2].

On the other hand, Antenna in Package (AiP) solutions exist for the $2.4 \mathrm{GHz}$ band [3], so there is significant direct coupling to a chip-size antenna. Moreover, with rising frequency, the bonding reactance rises too and the die starts to become more and more isolated from the IC pins: conducted interference will enter less and less well. For instance, a bond wire introducing $1 \mathrm{nH}$ of partial inductance means about $6 \mathrm{j} \Omega$ of series impedance at $1 \mathrm{GHz}$. Finally, radiated interference captured by PCB traces will increasingly suffer from loss with rising frequency. For instance, a typical transmission line loss of a trace on FR-4 substrate is $65 \mathrm{~dB} / \mathrm{m}$ at $20 \mathrm{GHz}$ [4]. This all suggests that the direct coupling to a chip-size circuit is not always negligible with respect to the conducted coupling in real-life context.

Therefore, this article will challenge the dominantconduction hypothesis, that is: the radiated immunity of PCBs can be sufficiently predicted up to several $\mathrm{GHz}$ by the fieldto-trace coupling and the conducted immunity of the IC only. In other words: we may neglect the direct coupling to the IC (i.e. the IC radiated immunity) up to several $\mathrm{GHz}$.

To that end, a case study will be performed, which will be drawn up in Section II. To predict its radiated immunity, two models are needed: one for field-to-trace coupling and one for the IC conducted immunity. The former will be recalled in Section III. The latter will be recalled and applied to the case study in Section IV. Both models will then be cascaded in Section V and compared to measurement results. Conclusions will be drawn upon these results in Section VI. The limitations of these conclusions will lead to the recommendations in Section VII. 


\section{CASE STUdy}

To study the dominant coupling mechanism of an incident field to a PCB equipped with an IC, a very simple and deterministic case had to be designed.

In order to illuminate a PCB with a deterministic field, a $10 \times 10 \mathrm{~cm}$ PCB was designed, suitable for a GTEM cell. It essentially consists of one straight, $50 \Omega, 5 \mathrm{~cm}$ microstrip trace. At one end, an SMA connector allows for placing arbitrary terminal loads. At the other end, an SOIC footprint allows for soldering a small IC.

For simplicity, a basic and common IC was chosen: the LM7805 linear voltage regulator. It has one input and one output and requires no peripheral components. This voltage regulator rectifies Continous Wave $(\mathrm{CW})$ disturbances on its input voltage to a DC offset on its output voltage. For simplicity, we will just consider the IC to 'pass' or to 'fail'. An output offset in excess of $\pm 100 \mu \mathrm{VDC}$ over a $1 \mathrm{k} \Omega$ load will be considered a failure. This extremely severe criterion was chosen in order to observe susceptibility with the experimentally available field strength.

The resulting case study is schematically depicted in Figure 1a. If the dominant-conduction hypothesis is true, its radiated immunity can be predicted with the field-to-trace coupling and the conducted immunity of the IC only, as illustrated in Figure $1 \mathrm{~b}$.

\section{Field-To-TRace Coupling Model}

The coupling of an incident plane wave to a PCB at the left of Figure 1b will be modeled with the Single Modified Taylor cell [5]. To allow for comparison with measurement on a GTEM cell, a simple model for the GTEM's imperfections will be added [6].

The Single Modified Taylor cell bears its name, because the modification allows modeling an electrically long line with a single cell. Taylor's original model for field-to-line coupling

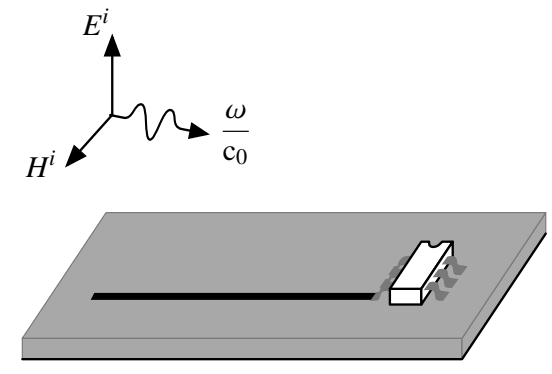

(a) Perspective on the system.

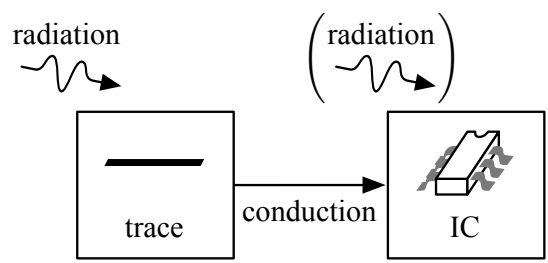

(b) Dominant-conduction model of the system: the radiated immunity of the IC can be neglected.

Figure 1. Illumination of the case study: a trace connected to an IC.

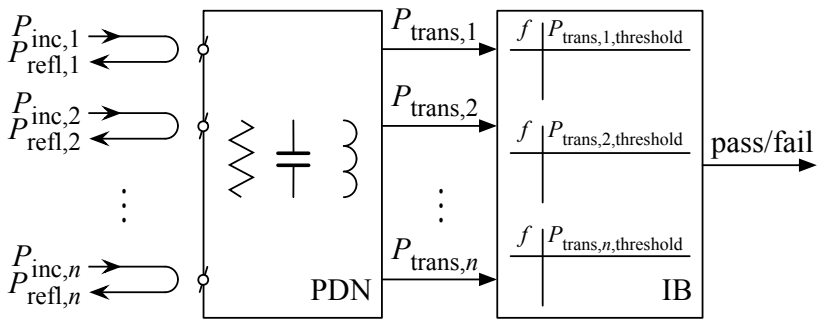

Figure 2. Symbolic representation of the ICIM-CI (IEC 62433-4).

[7] needed to be meshed along the line. Each cell would thus experience a locally uniform incident field. The coupling of all cells then needed to be added up numerically. For the case of a vertically polarized, grazing plane wave incident on a microstrip trace with characteristic loads, it has been shown that a single cell may suffice, modified by a correction factor for long line effects [8]. Basic transmission line theory can then by applied to take into account arbitrary loads [5].

The GTEM cell that will be used for measurements does not generate a perfect plane wave. A part of its imperfection can be understood as the residual reflection from its (imperfect) absorbers. The reflection can be quantified by measuring the $S_{11}$-parameter of the GTEM cell. To get a better prediction of the measurement, the PCB will therefore be considered to be illuminated twice: once by the nominal plane wave, and once by the residual reflection from the absorbers. The Modified Taylor cell will thus be used to calculate the coupling of the nominal field to the trace, then of the reflected field to the trace, and then both results will be added up. In this summation, the complex phase of the reflection at the PCB position determines whether constructive or destructive interference will occur. If the PCB position (hence the complex phase) is not known, the $S_{11}$-parameter still allows to calculate an uncertainty interval on the field-to-trace coupling [6].

\section{IC Conducted ImMunity Modeling}

Models can be thought as a model structure filled with parameters. Different model structures to describe the conducted immunity of integrated circuit have been proposed, but the most mature to date is ICIM-CI [1].

First, the ICIM-CI model structure will be outlined. Then, existing measurement methods to extract the model parameters from measurements will be presented and applied to the case study of this paper.

\section{A. ICIM-CI Model Structure}

An ICIM-CI predicts functional failure of an IC under CW disturbances entering the IC pins. To do so, it consists of a Passive Distribution Network (PDN), which predicts how much incident power is transmitted to the IC pins and an Immunity Behaviour (IB) part, which predicts whether this transmitted power will make the IC fail, as shown in Figure 2.

The PDN is a linear multiport, of which every port represents a ground-referenced IC pin. Using the PDN, the reflected and transmitted power can be calculated from a given incident power. The PDN can be described by $S$-parameters or by 
any network that can be linearized, such as an I/O Buffer Information Specification (IBIS) model.

The IB commonly consists of a look-up table for every port that yields a failure threshold as function of the disturbance frequency. If the disturbance on any port exceeds the threshold for the respective port, the IC is predicted to fail. Typically, this failure threshold is stored in the form of a transmitted power threshold.

If detailed information of the to-be-modeled IC is available, PDN and IB can be extracted from full-wave package and circuit simulations, for example. As this is neither our case, nor the typical case of PCB designers, the PDN and IB will be extracted from measurements on the IC [9].

\section{B. PDN Extraction by VNA Measurement}

To obtain the PDN, the $S$-parameters of an IC can be measured using a Vector Network Analyzer (VNA). The main challenge then is to move the reference plane to the IC pins. To that end, the IC was put on a solderless IC fixture. This fixture just consists of SMA connectors leading to the IC pins through $50 \Omega$ grounded coplanar waveguides (GCPWs). All feeds are made electrically equivalent and are faithfully reproduced on a calibration kit [10]. By calibrating with ideal standard definitions on this calibration kit, the reference plane can be moved to the IC pins.

To get the LM7805 IC into its operating point, $10 \mathrm{~V}$ was applied to the input through the VNA's bias tee. To check the solderless connections at DC, the output voltage was verified to be $5 \mathrm{~V}$ through the VNA's other bias tee. All unused and ground pins of the IC were shorted using a silver conductive pen to nearby ground vias $(<1 \mathrm{~mm})$. The measurement set-up is schematized in Figure 3a. The impedance of the IC's power input is measured, and after calibration with the calibration kit, it is shown in Figure 3b. This complex impedance constitutes the extracted PDN.

\section{IB Extraction by DPI Measurement}

The failure threshold can be measured using Direct Power Injection (DPI) [11]. This method consists of increasing the incident power upon the IC's pin under test until the IC fails. At this point of failure, the transmitted power is measured by subtracting incident and reflected power, both measured with a directional coupler. This value is then stored in the IB look-up table.

As shown by Lafon et al., this transmitted power measurement is particularly uncertain for highly reflective IC's [12]. Therefore, another method was used to deduce the transmitted power, illustrated in Figure 4a. Only the generator power threshold was recorded during the DPI measurement, i.e. the power incident on reference plane I. By calibration beforehand with a wattmeter, the gain of the amplifier and losses in cabling are known and the power incident upon the input bias tee (i.e. reference plane II) can be calculated. The insertion loss of the input bias tee was measured with a VNA and was subtracted to obtain the power incident upon the fixture connectors (i.e. reference plane III). Finally, the reflection tracking loss of the SOIC8 fixture feed was obtained by offline calibration [10]

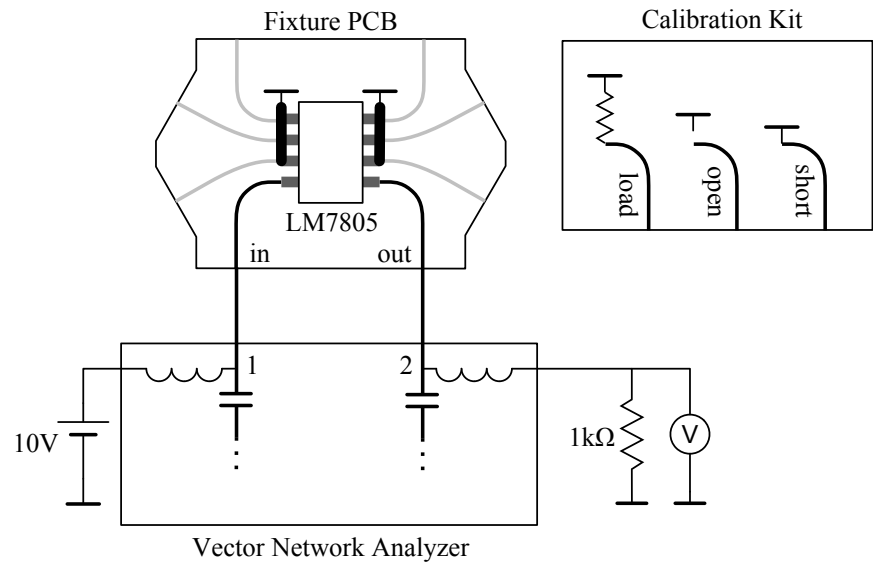

(a) Set-up.

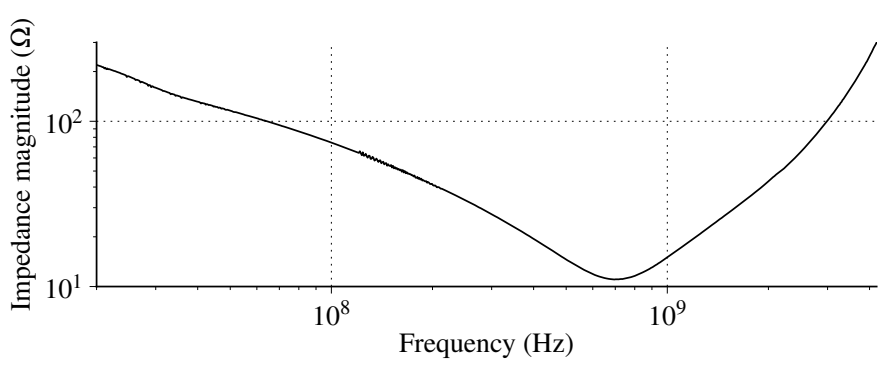

(b) Results.

Figure 3. PDN extraction by VNA measurement on the LM7805 IC.

and subtracted to know the power incident upon the IC pins (i.e. reference plane IV).

To speed up the DPI measurement, successive approximation was used: the failure threshold was sought by stepping the generator power in steps of $5,1,0.5$ and $0.25 \mathrm{~dB}$ [13]. After each refinement, the power was lowered to encounter failure while stepping $u p$, in order to avoid hysteresis uncertainty. This way, the incident power threshold at the IC pin $P_{\text {inc,th }}$ was measured with a $0.25 \mathrm{~dB}$ resolution, which is rather precise.

Because of the severe criterion $( \pm 100 \mu \mathrm{VDC})$, the nondisturbed voltage drift during the DPI measurement could not be neglected. Therefore, before every frequency-power point, the RF disturbance was turned off and the nominal output voltage was measured again. Probably, this drift occurred due to heating of the IC, but the root cause of this heating is unknown (environment or RF power).

Using the input reflection coefficient $S_{11}$ of the IC, the transmitted power threshold $P_{\text {trans,th }}$ can be calculated from the incident power threshold $P_{\text {inc,th }}$ :

$$
P_{\text {trans,th }}=P_{\text {inc,th }} \cdot\left(1-\left|S_{11}\right|^{2}\right) .
$$

Alternatively, the failure threshold can be expressed in terms of voltage:

$$
V_{\mathrm{th}}=2 \sqrt{P_{\text {inc,th }} Z_{c}} \frac{Z_{11}}{Z_{11}+Z_{c}} .
$$

The failure threshold, which constitutes the IB, is plotted in both forms in Figure $4 b$. 


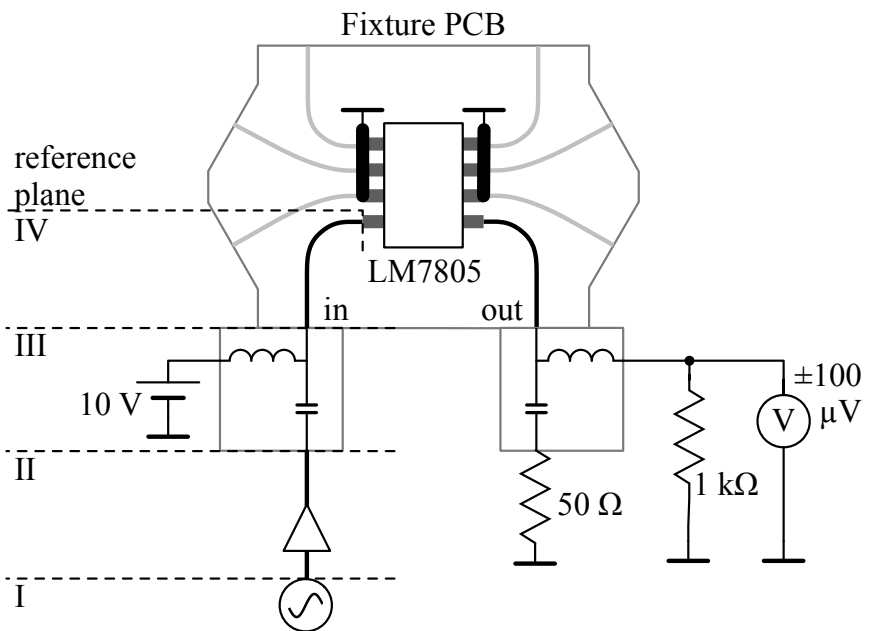

(a) Set-up.
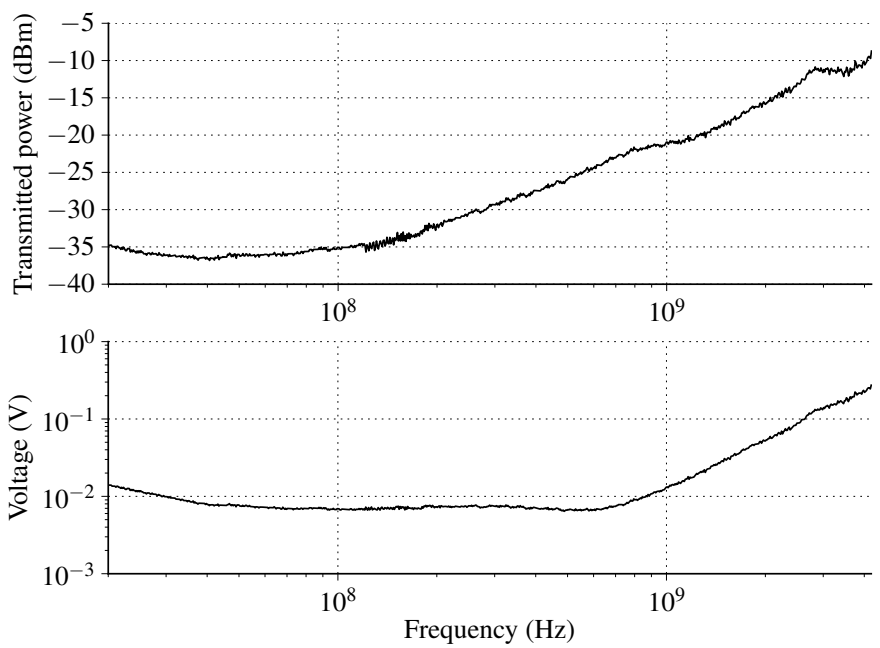

(b) Resulting failure threshold in two forms: transmitted power and voltage.

Figure 4. IB extraction by DPI measurement on the LM7805 IC for a $\pm 100 \mu \mathrm{VDC}$ output failure criterion.

\section{Cascading Trace and IC}

Now that the field-to-trace coupling can be calculated and the conducted immunity of the IC is known, the radiated immunity of the whole can be predicted, as illustrated in Figure $1 b$.

Two configurations were predicted and measured. Firstly, an end-fire illuminated trace with the IC at the far end. The nearend was terminated with a broadband $50 \Omega$ load, as illustrated in Figure 5b. Secondly, a very different configuration was created within constraints of the set-up. The card was rotated half a turn; the trace is still end-fire illuminated, but the IC is now located at the near end. To challenge the model on reflections on the trace, the far-end $50 \Omega$ load was exchanged for a short calibration standard.

The first step is to calculate the field-to-trace coupling for each configuration. One end of the trace is terminated by the IC's impedance as measured in Section IV-B. The other end is terminated by the PCB transition to the SMA reference plane, modeled as a lossless $48.2 \mathrm{ps}$ delay [14], followed by the supply bias tee. The reflection coefficient of the bias tee

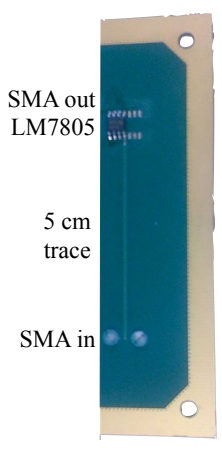

(a) Inner side.

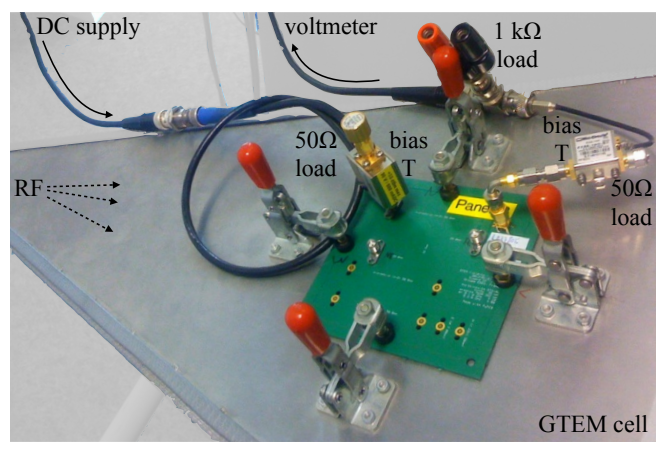

(b) Outer side, PCB placed on the GTEM cell.
Figure 5. Demonstrator PCB for radiated immunity of trace and IC.

was measured with a matched load and the short standard, respectively, to model both configurations. The measured reflection coefficient of the GTEM cell was measured and taken into account as explained in [6], to calculate the voltage transfer $H$ from the septum to the IC pin.

The next step is to divide the failure threshold voltage $V_{\text {th }}$ by the voltage transfer $H$, to obtain the septum threshold voltage $V_{\text {th,septum. }}$ Finally, the threshold power incident on the GTEM cell input is:

$$
P_{\text {inc,GTEM,th }}=\frac{V_{\text {th,septum }}^{2}}{Z_{c, \text { GTEM }}}=\frac{\left(V_{\mathrm{th}} / H\right)^{2}}{50.0} .
$$

These formulæ were evaluated using Python. The scikit-rf library was used to perform offline calibration and reference plane shifts [15].

The radiated immunity in the GTEM cell was measured similarly to the DPI, that is, with $0.25 \mathrm{~dB}$ incident power resolution and by measuring the undisturbed output voltage before each power-frequency point. RF amplifiers were used to obtain the necessary power; our amplifiers had a bandwidth of $20 \mathrm{MHz}$ to $4.2 \mathrm{GHz}$. Their integrated directional couplers were used to measure the power incident upon the GTEM cell input. The directional couplers were calibrated to the GTEM cell input. The results are compared with the respective predictions in Figure 6.

The peak in the measurement at $290 \mathrm{MHz}$ occurred intermittently, both in DPI and GTEM measurements. Probably, the voltage regulator without external output capacitor is marginally stable around this frequency, and the disturbance may excite this oscillation. Notice also how the GTEM measurement flattens around the alleged $+46 \mathrm{dBm}(40 \mathrm{~W}$ !) at low frequencies. One must be careful when interpreting this number, which is derived from measurements with diode-based average power meters connected to the directional couplers of the amplifiers. The amplifier used in this range has an output $3 \mathrm{~dB}$ compression point at $25 \mathrm{~W}$, so the $40 \mathrm{~W}$ is the sum of the disturbance frequency and its harmonics, which are significant in power. Consequently, for these high powers, the meaning of the measured curve is not purely the immunity against one frequency. However, this is the meaning of the predicted curve. Therefore, this might explain the divergence of prediction and measurement. 


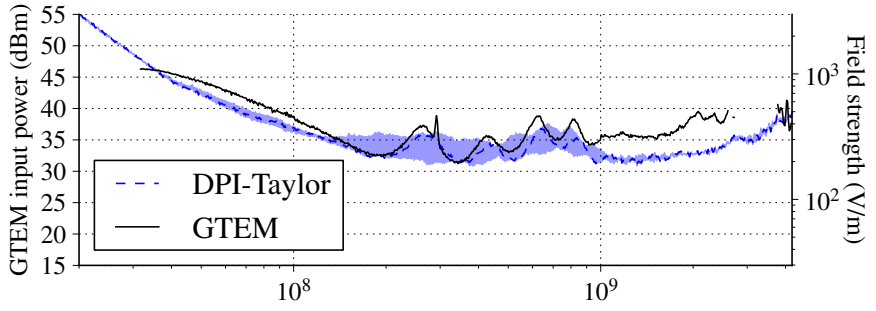

(a) Near end: matched load. Far end: LM7805 input.

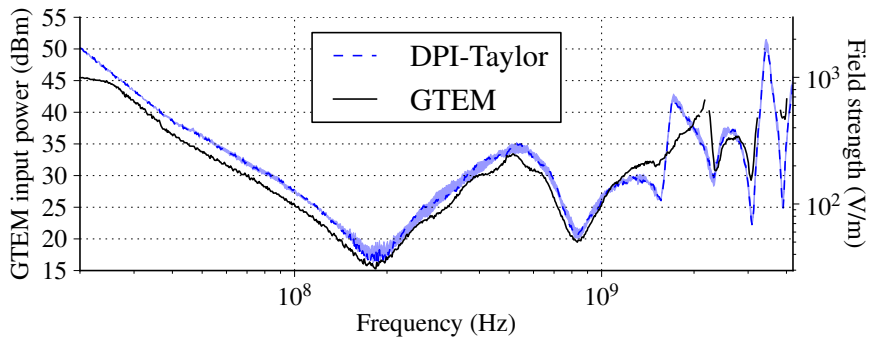

(b) Near end: LM7805 input. Far end: short standard.

Figure 6. Prediction and measurement of the radiated immunity of a $5 \mathrm{~cm}$ trace and an LM7805 voltage regulator. The shaded area represents the uncertainty interval if the PCB position with respect to the GTEM absorbers would not be known. Gaps in the black curve signify that no susceptibility could be observed with the available RF power.

\section{CONCLUSions}

In this paper, the dominant-conduction hypothesis was formulated: up to $10 \mathrm{GHz}$, interference enters SOIC packages predominantly by conduction. To challenge this hypothesis, the existing Modified Taylor model for field-to-trace coupling and an ICIM-CI model were concatenated to predict the radiated immunity of the combination of a $5 \mathrm{~cm}$ PCB trace and an LM7805 voltage regulator IC. Two different configurations were measured: a short circuit and a matched load at the other end of the trace. Although the radiated immunity of the IC itself was thus neglected, good correlation between measurement and prediction was observed up to $4.2 \mathrm{GHz}$. Above this frequency, the necessary power to observe failure was not available. Although we would have liked to measure until $10 \mathrm{GHz}$, at least this observation did not falsify the hypothesis.

At least two critical remarks may be made. Firstly, it may not come as a surprise that the coupling to a $5 \mathrm{~cm}$ PCB trace dominates over the coupling to a subcentimeter IC. In modern electronics, PCB traces may be as small as IC packages. Secondly, one may also object that the $\pm 100 \mu$ VDC definition of 'failure' is ridiculously severe, and that the radiated immunity issues of modern-day devices have other root causes.

\section{RECOMMENDATIONS}

Both critical issues mentioned above may be tackled by taking a real-life, non-compliant case study. If the method presented in this paper succeeds in predicting its non-compliance, we may gain trust in the dominant-conduction hypothesis.

Ideally, the cost of non-compliance, redesign and a new prototyping cycle should be known in this case study. If the cost of modeling and simulation is estimated, too, the Return
On Modeling Effort (ROME) may be quantified. This will steer future research towards results with an industrial, reallife impact.

In parallel, industrial impact could be made by putting the field-to-trace coupling and IC immunity models in the hands of PCB designers. They typically do not have the time to read scientific papers, or to adapt them to their workflow. A push-of-the-button tool should therefore be elaborated, that integrates with their Electronic Design Automation (EDA) tools $[17,18]$. Supplying this kind of tools will also yield useful feedback from the industry.

If through all this, conduction remains the dominant interference mechanism for integrated circuits above $1 \mathrm{GHz}$, Gigahertz DPI (GDPI) measurements are useful. To that end, the IEC 62132-4 standard should be extended to prescribe and propose measurement set-ups that are valid beyond $1 \mathrm{GHz}$. As the wavelength approaches the size of IC pins, the definition of the reference plane of ICs and the incident Quasi-Transverse Electromagnetic (QTEM) mode should be contemplated again, with consequences for IEC 62433-4 [16].

\section{ACKNOWLEDGEMENTS}

This research project was co-financed by the French national project SEISME (simulation of emissions and immunity of electronic systems). The authors thank Etienne Sicard for his GDPI vision, shared with researchers worldwide.

\section{REFERENCES}

[1] IEC 62433-4: EMC IC modelling - Part 4: Models of Integrated Circuits for RF immunity behavioural simulation - Conducted Immunity Modelling (ICIM-CI). IEC, 2016.

[2] Jorge L. Lagos and Franco L. Fiori. "Worst-Case Induced Disturbances in Digital and Analog Interchip Interconnects by an External Electromagnetic Plane Wave - Part I: Modeling and Algorithm". In: Electromagnetic Compatibility, IEEE Transactions on 53.1 (Feb. 2011), pp. 178-184. ISSN: 0018-9375. DOI: 10.1109/TEMC.2010.2085005.

[3] Antenna in Package Design Methodology. 2010. URL: http://www. insightsip.com/images/stories/download/technical_documentation/aip_ methodology_whitepaper_isip.pdf.

[4] A. Rajagopal. "Printed circuit board (PCB) loss characterisation up to $20 \mathrm{GHz}$ and modeling, analysis and validation". PhD thesis. University of Missouri-Rolla, 2007.

[5] Sjoerd T. Op 't Land et al. "Field-To-Long-Segmented-Trace Coupling with Arbitrary Loads and a Transparent Upper Bound Using a Single Modified Taylor Cell”. In: IEEE Transactions on Electromagnetic Compatibility 58.5 (July 2016), pp. 1517-1525. DOI: 10.1109/TEMC. 2016.2566449

[6] Sjoerd T. Op 't Land, Richard Perdriau, and Mohamed Ramdani. "Taking Into Account GTEM Field Nonuniformities in Radiated Immunity Simulations Through a Simple Measurement". In: IEEE Transactions on Electromagnetic Compatibility PP.99 (2017), pp. 1-4. ISSN: 00189375. DOI: 10.1109/TEMC.2017.2691800.

[7] Clayborne D. Taylor, R.S. Satterwhite, and Charles W. Harrison Jr. "The response of a terminated two-wire transmission line excited by a nonuniform electromagnetic field". In: Antennas and Propagation, IEEE Transactions on 13.6 (Nov. 1965), pp. 987-989. ISSN: 0018926X. DOI: 10.1109/TAP.1965.1138574.

[8] Sjoerd T. Op 't Land et al. "Simple, Taylor-based worst-case model for field-to-line coupling". In: JPIER 140 (June 2013), pp. 297-311. DOI: $10.2528 /$ PIER13041207.

[9] Frédéric Lafon et al. "An Industry-Compliant Immunity Modeling Technique for Integrated Circuits". In: 2009 Kyoto International Symposium on EMC. July 2009.

[10] Sjoerd T. Op 't Land, Richard Perdriau, and Mohamed Ramdani. "Design of a $20 \mathrm{GHz}$ DPI method for SOIC8". In: Electromagnetic Compatibility (EMC EUROPE), 2012 International Symposium on. Sept. 2012, pp. 1-6. DOI: 10.1109/EMCEurope.2012.6396691. 
[11] IEC 62132-4 Integrated circuits - Measurement of electromagnetic immunity $150 \mathrm{kHz}$ to $1 \mathrm{GHz}$ - Part 4: Direct $\mathrm{RF}$ power injection method. IEC, Sept. 2005.

[12] Frédéric Lafon et al. "Extending the Frequency Range of the Direct Power Injection Test: Uncertainty Considerations and Modeling Approach". In: EMC Compo 2009. Nov. 2009.

[13] Jean-Baptiste Gros, Geneviève Duchamp, and Jean-Luc Levant. "A Immunity Measurement Bench Dedicated to Analog To Digital Converter". In: EMC Europe 2010, 9th International Symposium on EMC and 20th International Wroclaw Symposium on Electromagnetic Compatibility. Wroclaw, Poland: Oficyna Wydawnicza Politechniki Wroclawskiej, Sept. 2010, pp. 685-690.

[14] Sjoerd T. Op 't Land et al. "Using a Modified Taylor Cell to Validate Simulation and Measurement of Field-to-Shorted-Trace Coupling". In: IEEE Transactions on Electromagnetic Compatibility 56.4 (2014), pp. 864-870. ISSN: 0018-9375. DOI: 10.1109/TEMC.2014.2313231.

[15] scikit-rf, Object Oriented RF Engineering. URL: http ://scikit - rf . readthedocs.io.

[16] Sjoerd T. Op 't Land. "Integrated circuit immunity modelling beyond 1 GHz". Theses. INSA de Rennes, June 2014. URL: https://tel.archivesouvertes.fr/tel-01165061.

[17] Ala Ayed et al. "FastImmunity: An EDA extension for PCB immunity prediction". In: 2016 Asia-Pacific International Symposium on Electromagnetic Compatibility (APEMC). Vol. 01. May 2016, pp. 207-210. DOI: 10.1109/APEMC.2016.7523011.

[18] FastImmunity: predicting PCB radiated immunity from within an EDA tool. Screencast. URL: https://vimeo.com/182572446. 